\title{
Convergence P2P context awareness
}

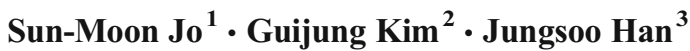

Published online: 2 November 2015

(C) Springer Science+Business Media New York 2015

Welcome to this special issue of Peer-to-Peer Networking and Applications on "Convergence P2P Context Awareness," which contains fifteen of the best papers submitted to the issue. The purpose of this special issue is to publish current and relevant research papers from academia and practitioners in various industries on this emerging topic.

In the IT convergence environment, various sensors and computing resources, including mobile devices and networks in users' everyday lives, should be aware of the users' intentions and the surrounding environment and should provide services based on that awareness. Providing personalized services for users requires convergence peer-to-peer (P2P) context awareness that can efficiently share various forms of information collected by computing resources ubiquitous in users' daily spaces, and that can automatically recognize and process context information about users and their surrounding environments [14-16]. This special issue covers some of the hottest topics in Convergence P2P Context Awareness,

Jungsoo Han

jungsoo.han.k@gmail.com

Sun-Moon Jo

sunmoon@pcu.ac.kr

Guijung Kim

gikim@konyang.ac.kr

1 Department of Computer Information Technology Education, Paichai University, Doma 2-dong, Seo-gu, Daejeon, Republic of Korea

2 Department of Biomedical Engineering, Konyang University, 121, Daehak-ro, Nae-dong, Nonsan-si, Chungcheongnam-do, Republic of Korea

3 Division of Information \& Communication, Baekseok University, 76, Munam-ro, Anseo-dong, Dongnam-gu,

Cheonan-si, Chungcheongnam-do, Republic of Korea including: Hybrid P2P networking system; P2P Knowledge based Applications and Management; P2P Multicasting and multimedia delivery; P2P Overlay networks; P2P Security issues; P2P Wireless and mobile networks; P2P Data and index structures; P2P Modeling, performance, and robustness; Semantic routing and search in $\mathrm{P} 2 \mathrm{P}$; P2P Architectures and protocols; Convergence/co-existence of P2P and other networks.

The paper, by Ji et al. [1]., proposes mining students activities from a computer supported collaborative learning system based on peer to peer network. Our proposed method is a learner activity analysis model based on learner activity information generated in a computer-supported collaborative learning (CSCL) system that students, teachers, and administrators can use to conduct a learner activity analysis that is easy to understand and effective. As for the proposed learner activity model, it classifies learning activities in a CSCL system into three categories (vivacity, learning, and relationship) and then creates quotients to represent them accordingly. The paper, by Kim et al. [2]., proposes the efficient peer-to-peer context awareness data forwarding scheme based on the devices location in emergency situations. This efficient P2P data forwarding scheme (EP-DFS) can be applied to various fields that are needed for $\mathrm{P} 2 \mathrm{P}$ communications and networking. To support P2P transmission in the proposed EP-DFS, each intermediate device located in the transmission path establishes a synchronized path. The user can communicate with other people, and relay the message to the outside world in emergency mode.

The paper, by Li et al. [3]., shows the effects of social media usage on country image and purchase intention from social P2P network perspective. The proposed method examines the impact of social media usage on a country's image, and the relationship between social media usage and the images of a country's economy, politics, nationality, and culture. 
This paper also examines the impact of country image on product image and purchase intention. The method reveals these relationships from a social P2P network perspective that also provides new insights into the effects between these variables in order to provide measures for improving the national image. The paper, by Jung et al. [4]., proposes a security monitoring method for malicious P2P event detection. P2P file sharing events were obtained and the behavior analyzed to compensate for defects. The proposed system can detect suspicious P2P sharing and synchronize with a security monitoring system on a virtual machine. The characteristics can be applied for security monitoring to block and respond to the distribution of malicious code through P2P.

The paper, by Chae et al. [5]., proposes a privacy data leakage prevention method in P2P networks. The proposed method can be used as a privacy data leakage prevention method by releasing a P2P sharing file that does not include privacy data using privacy data-removing technology with a privacy data-leaking risk factor. This process solved the issues of data loss prevention (DLP) systems, such as a high detecting error with privacy data and invasion of privacy issues. The paper, Joh et al. [6]., introduces the Internet of everything based on energy efficient P2P transmission technology with Bluetooth low energy. The proposed method is an energy-efficient hybrid P2P transmission technology for P2P networking environments, and the authors show that the proposed scheme can minimize the initial connection-establishment delays that are inevitable in existing Wi-Fi P2P data transmission technology. This technology can be applied not only to the peer-to-peer networking environment but also to the design and implementation of an Internet of Things (IoT) infrastructure where all fixed and mobile devices are connected with no limitation.

The paper, by Yang et al. [7]., proposes mVoIP for P2P service based authentication system using AA authentication server. The proposed method is a system for addressing security vulnerabilities from the increase in the use of voice over Internet protocol (VoIP) services and for providing differentiated services according to user access privileges. An authentication system was designed by adding the AA Server to the existing VoIP session configuration stage to provide differentiated services according to user access, and to add security. The paper, by Bae [8]., presents the designing and verifying of a P2P service security protocol in M2M environment. The proposed protocol is kept safe against a variety of intruder attacks by requiring formal verification using a compiler for the analysis of security protocols (Casper) and failure divergence refinements (FDR). This draws on hash functions, real-time secret agents and encryption key values and inserts a session key and public key so as to transmit optimized values that vary each session.

The paper, by Jung et al. [9]., purposes a P2P context awareness based sensibility design recommendation using the color and bio-signal analysis. The proposed method is P2P network-based collaborative filtering that uses a rules-based, context-aware, cluster, P2P network, as well as learning where the user has already evaluated preferences for recommendation. This study analyzes the relation between visual sensibility and color design with the use of statistical analysis tools, and the clustering of users with similar sensibilities is conducted with the use of P2P network-based context awareness. The paper, by Cha et al. [10]., shows a node management scheme for stable P2P service in mobile Ad-hoc networks. Under the proposed scheme, nodes that have temporary defects but that are otherwise normal and can be recovered, are kept in the network; but those that are defective or malicious are eliminated from the network using trust values. Network reliability was improved by detecting malicious nodes, although there is no regular management of the detected nodes, and it is difficult to make predictions about mobility.

The paper, by Chung et al. [11]., proposes P2P cloud network services for IoT based disaster situations information. The proposed service combines an IoT/M2M network with a P2P cloud service for a rapid and smooth response in the event of a disaster, and provides the results via social services such as SNS. To this end, a wide area wireless disaster information network system has been built locally, and each local network is connected to the other to deal with disaster situations by using the server of the disaster area. The paper, by Yang et al. [12]., proposes hybrid trust evaluation model for identifying malicious behavior in mobile P2P. The proposed method is a hybrid trust evaluation technique based on a trust zone structure to improve reliability between nodes. A Trust Zone Manager (TZM) node is elected for trust evaluation of member nodes internal to each trust zone. The certificates of member nodes are issued in the elected TZM, and the information is stored in a Trust Zone Member Table (TZMT). The paper, by Kim et al. [13]., proposes P2P-based u-health cluster service model for silver generation in PBR platform. The proposed service provides health, dietary and exercise information regardless of time and space on a PBR(Personal Bio Record) platform. The developing system effectively connects to EMR(Electronic Medical Record) and users' service systems in order to understand the service targets' lifestyle patterns and to offer further customized services. This model is expected to provide useful services to users in a multi-platform 
environment and contribute to enhanced health status of senior patients with chronic disease.

This fine collection of papers was achieved by fruitful collaborations. We wish to thank all the authors for their contributions and the reviewers for assisting our editorial work. We do hope the papers included in this issue will satisfy the audience of Peer-to-Peer Networking and Applications and readers will find the papers interesting. Furthermore, we would like to thank Professor Sherman Shen, editor-in-chief of Peer-to-Peer Networking and Applications, for his valuable remarks and his undeterred help throughout the publication process of this special issue.

\section{References}

1. Ji H, Park K, Jo J, Lim H (2015) Mining students activities from a computer supported collaborative learning system based on peer to Peer Network. Peer-to-Peer Netw Appl. doi:10.1007/s12083-0150397-0

2. Kim S, Suk J (2015) Efficient peer-to-peer context awareness data forwarding scheme in emergency situations. Peer-to-Peer Netw Appl. doi:10.1007/s12083-015-0401-8

3. Li G, Park E-M, Park S-T (2015) Effects of social media usage on country image and purchase intention from social P2P network perspective. Peer-to-Peer Netw Appl. doi:10.1007/s12083-015-0379-2

4. Jung HM, Hwang I-S, Moon J-K, Park H-S (2015) A security monitoring method for malicious $\mathrm{P} 2 \mathrm{P}$ event detection. Peer-toPeer Netw Appl. doi:10.1007/s12083-015-0369-4

5. Chae C-J, Shin YJ, Choi K, Kim K-B, Choi K-N (2015) A privacy data leakage prevention method in P2P networks. Peer-to-Peer Netw Appl. doi:10.1007/s12083-015-0371-x

6. Joh H, Yang I, Ryoo I (2015) The internet of everything based on energy efficient P2P transmission technology with Bluetooth low energy. Peer-to-Peer Netw Appl. doi:10.1007/s12083-015-0377-4

7. Yang H-K, Cha H-J, Kim Y-H (2015) MVoIP for P2P service based authentication system using AA authentication server. Peer-to-Peer Netw Appl. doi:10.1007/s12083-015-0394-3

8. Bae W-S (2015) Designing and verifying a P2P service security protocol in M2M environment. Peer-to-Peer Netw Appl. doi:10. 1007/s12083-015-0396-1

9. Jung H, Chung K (2015) P2P context awareness based sensibility design recommendation using color and Bio-signal analysis. Peerto-Peer Netw Appl. doi:10.1007/s12083-015-0398-Z

10. Cha H-J, Yang H-K, Kim Y-H (2015) A node management scheme for stable P2P service in mobile ad-hoc networks. Peer-to-Peer Netw Appl. doi:10.1007/s12083-015-0392-5

11. Chung K, Park RC (2015) P2P cloud network services for IoT based disaster situations information. Peer-to-Peer Netw Appl. doi:10.1007/s12083-015-0386-3

12. Yang H-S, Sun J-H (2015) A study on hybrid trust evaluation model for identifying malicious behavior in mobile P2P. Peer-to-Peer Netw Appl. doi:10.1007/s12083-015-0411-6

13. Kim JT, Pan H-J, Kim J (2015) P2P based u-health cluster service model for silver generation in PBR platform. Peer-to-Peer Netw Appl. doi:10.1007/s12083-015-0387-2
14. Jung H, Chung K (2015) Sequential pattern profiling based Biodetection for smart health service. Clust Comput 18(1):209-219

15. Kim S-H, Chung K (2015) Emergency situation monitoring service using context motion tracking of chronic disease patients. Clust Comput 18(2):747-759

16. Jo SM, Chung K-Y (2015) Design of access control system for telemedicine secure XML documents. Multimed Tools Appl 74 (7):2257-2271

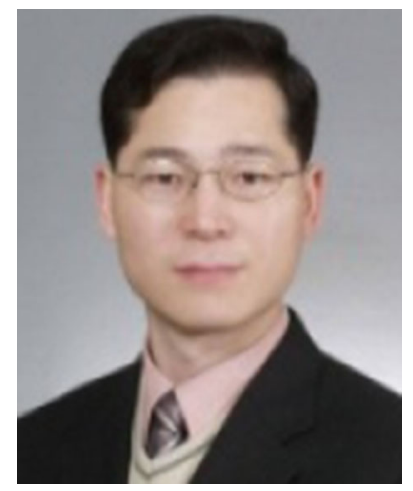

Sun-Moon Jo received the Ph.D. degrees from the Inha University, Korea, in 2007. He has worked for Seven System Korea. He is currently a professor in the Department of Computer Information Technology Education, Paichai University, Korea. His research interests include XML, Security, Knowledge System, HCI, Deep learning, and Recommendation. He serves as Director and is a member of the Editorial Committee of the Korea Knowledge Information Technology Society, as Director of the Korea Contents Association, as Member of the Korean Society for Internet Information, as Member of the Korea Information Processing Society, as Member of the Institute of Electrical and Electronics Engineers, as Steering Committees of International Conference on Digital Policy \& Management, and as Excellent Professor Award of the August 2015 Paichai University President. Also, he is an editorial board member of several International Journals.

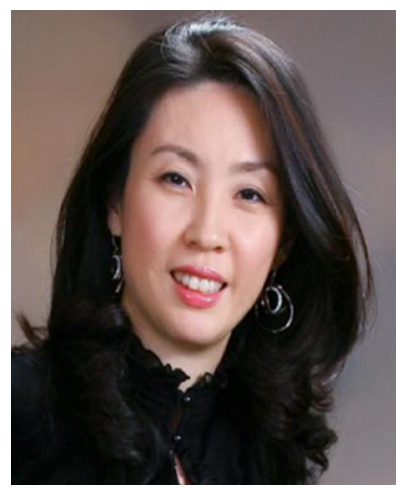

Guijung Kim received the B.S. degree and the M.S. degree in computer engineering from Hannam University, Republic of Korea, and the Ph.D. degree in computer engineering from Kyunghee University, Republic of Korea in 2003. Since 2001, she has been a professor in department of Biomedical Engineering, Konyang University, Chungnam, Korea. Her research interests include computer architecture, information retrieval, e-Learning, u-healthcare, security, data mining, and HCI. She serves as Executive Editing Director, Steering Committees of International Conference on Convergence Technology, Steering Committees of International Conference on Digital Policy \& Management. Also, she is an editorial board member of several International Journals. 


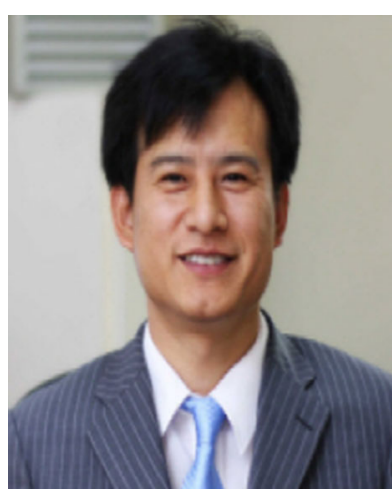

Jungsoo Han received a BS, an $\mathrm{MS}$, and a $\mathrm{PhD}$ in Computer Engineering from Kyung Hee University, Republic of Korea. Since 2001, he has been a Professor in the Division of Information \& Communication, Baekseok University, Cheonan City, Chungnam, Republic of Korea. In 2014, he researched Convergence IT and Creative Education Methodology at California State University Fullerton as an Exchange Professor. His research topics include Data Mining, Contents Planning, 3D

Modeling and CBD, Telemedicine, Knowledge-based Decision Support Systems, Intelligent Systems, Convergence, HCI, and Recommendation
Systems. He has edited books on computer science and convergence technology. He serves as Executive Editing Director of the International Conference on Convergence Content (ICCC), as General Co-Chair of the International Conference on Digital Policy \& Management (ICPDM), as General Co-Chair for steering committees of the International Conference on Convergence Technology (ICCT), as Workshop Chair of the International Conference on Information Science and Application 2013, as Workshop Chair of the $2^{\text {nd }}$ International Conference on IT Convergence and Security 2012, as Vice President of the Korea Contents Association, as Vice President and a member of the Editorial Committee of the Society of Digital Policy \& Management, and as Vice President of the Editorial Committee of the Korea Contents Association. 